\title{
Belief Function Theory in Constraint Satisfaction Problems: a Unifying Approach* $^{*}$
}

\author{
Aouatef Rouahi \\ ISG of Tunis, Tunisia \\ Kais Ben Salah \\ Khaled Ghedira \\ rouahi.aouatef@hotmail.fr \\ University of Jeddah \\ Central University, Tunisia \\ kaisuria@yahoo.fr \\ khaled.ghedira@isg.rnu.tn
}

\begin{abstract}
The Constraint Satisfaction Problem (CSP) is acknowledged as a simple declarative formalism for modeling welldefined decision problems. However, real-world problems are usually ill-defined, especially, under uncertain circumstances. In such situation, uncertainty evokes the need for flexibility or softness where we accept satisfying some constraints to some degree. Moreover, when the relevance of some constraints depends on other factors, we should prioritize those constraints. Eventually, the modeled uncertainty, as well as the expressed soft and prioritized constraints induce preferences over the solutions set. Previous work employing mathematical uncertainty theories are either uncertainty-based frameworks or preference-based ones and the only attempt to handle both uncertainty and preferences is performed using two uncertainty theories under a commensurability assumption. In this paper, we propose a unifying CSP extension, labeled Belief CSP, that deals jointly with all these four concepts, i.e., uncertainty, soft and prioritized constraints and preferences over the solutions set, by exploiting the expressiveness of the belief function theory.
\end{abstract}

\section{Introduction}

The classical Constraint Satisfaction Problem (CSP) $[6,5]$ framework has carried high attention because of its simplicity and generality. In fact, every problem that can be described by a set of variables and a set of constraints among those variables can easily be cast as a CSP

However, decision problems tackled by the classical CSP are assumed to be well-defined so that all their features are precise and known with certainty. Hence, the CSP has proven unfit for reasoning under uncertainty where most of the problems are ill-defined, i.e., some problem's components are either beyond our control or cannot be assimi-

\footnotetext{
*10.18293/SEKE2018-133
}

lated due to the lack of the required quantity and quality of knowledge or even ignorance.

From another side, yes-or-no reasoning makes no sense in uncertain contexts. In fact, uncertainty evokes the need for flexibility as the satisfaction of some constraints depends on ill-known components. In addition, the majority of the problems being solved are over-constrained, i.e., they do not lead to any solution. Therefore, it is necessary to soften some constraints in order to meet a solution, that is, accepting satisfying some constraints to some degree.

To boot, in real world problems, not all constraints are fully reliable. In other words, under uncertainty, the agent cannot entirely rely on some constraints that can be untrustworthy, or subject to change. This reliability affects the relevance of each constraint to the problem. Therefore, we should rank those constraints according to their reliability by assigning priorities.

As well, solutions cannot be equally preferable. In fact, uncertainty induces preference levels over the set of solutions.

In response to these issues, a variety of frameworks has been introduced. We are particularly interested in CSP extensions that employed mathematical uncertainty theories. Those extensions may be split in two trends: Uncertaintybased frameworks that focus on handling uncertainty in CSPs as suggested by the probabilistic CSP [4], which admits that the presence or the relevance of some constraints to the real problem may be uncertain.

Besides, the preference-based frameworks that focus on relaxing constraints in order to make an over-constrained problem solvable, such as the possibilistic CSP [10], which induces preference degrees over the constraints set to evaluate the necessity of the satisfaction of each constraint and the fuzzy CSP [3] that considers a constraint as a fuzzy set by making the satisfaction of a constraint by each labeling a matter of degree.

Yet, despite this variety of frameworks, there is no proposal that addresses the whole panel of those four notions mentioned above. We shall mention the work proposed in 
[2] that combined two theories, that are possibility theory and fuzzy sets theory, to express both uncertainty and soft constraints in CSPs but under a commensurability assumption between uncertainty degrees and satisfaction degrees.

In this paper, we introduce a unifying CSP framework, labeled Belief Constraint Satisfaction Problem, that deals jointly with uncertainty, flexibility, priorities and preferences in CSPs employing one uncertainty theory, that is, the Belief Function Theory (BFT) [1, 7, 8], on account of its expressiveness. The BFT proposes a natural tool for imperfection ${ }^{1}$ modeling as it allows handling uncertain as well as imprecise data.

The paper is organized as follows: The next section presents a formal definition of the BCSP and shows how uncertainty is handled and which components are involved; it also illustrates the prioritized and soft constraints modeling and preferences expressing. In the section 3 we will apply the BCSP to a simple agricultural land-use planning problem followed by a discussion of the main contribution and some further work.

\section{Belief Constraint Satisfaction Problem}

\subsection{Preliminaries}

A classical CSP is defined by a quadruplet $(X, D, C, R)$ where $X=\left\{x_{1}, \ldots, x_{n}\right\}$ is a finite set of $\mathrm{n}$ variables, each $x_{i}$ takes its values in a finite domain $D_{i}$ such that $D=$ $\left\{D_{1}, \ldots, D_{n}\right\}$. The simultaneous assignment of values to a set of variables is called an instantiation and denoted by $\theta$. $C=\left\{C_{1}, \ldots, C_{m}\right\}$ is a finite set of $m$ constraints where each constraint $C_{i}$ is defined on a subset of variables $S_{i} \subseteq X$ delimited its scope and by a relation $R_{i}$ that specifies the set of permitted instantiations with respect to $C_{i} ; R_{i}$ is a subset of the Cartesian product of the domains of the variables in $S_{i}$ (i.e., $R_{i} \subseteq \times\left\{D_{i} \mid x_{i} \in S_{i}\right\}$ ). A solution of a CSP is a consistent complete instantiation, i.e., an instantiation of all the variables in $X$, so that, all the constraints in $C$ are satisfied. A CSP is said to be consistent if and only if it has at least one solution, otherwise, it is said to be inconsistent.

\subsection{Belief Constraint Satisfaction Problem (BCSP)}

A Belief Constraint Satisfaction Problem is a quadruplet $\left(X, D, C_{\alpha}, R\right)$, where:

- $X=\left\{x_{1}, \ldots, x_{n}\right\}$ is a finite set of variables;

- $D=\left\{D_{1}, \ldots, D_{n}\right\}$ is the set of their domains;

\footnotetext{
${ }^{1}$ The term uncertainty is commonly used, in the literature, to refer to imperfection. However, according to the taxonomy established in [9] uncertainty is one aspect of imperfection, whereas, imprecision is its other aspect.
}

- $C_{\alpha}=\left\{\left(C_{1}, \alpha_{1}\right), \ldots,\left(C_{m}, \alpha_{m}\right)\right\}$ is a finite set of belief constraints (shortened as Bf-constraints) where $(1-$ $\left.\alpha_{i}\right)$ is the priority of the $\mathrm{Bf}$-constraint $C_{i}$.

- $R=\left\{R_{1}, \ldots, R_{m}\right\}$ is a finite set of imperfect relations associated to $C_{\alpha}$.

\subsubsection{Imperfect Relations}

Under uncertainty, we cannot doubtlessly state whether or not an instantiation of values to variables is permitted with reference to a given constraint. To model imperfect (uncertain and/or imprecise) relations, we rely on a basic belief mass (bbm) distribution over instantiations.

An imperfect relation $R_{i}$, defined by a pair $\left(V_{i}, \Theta_{i}\right)$, associates a valuation $V_{i}$ (a bbm distribution), over the frame of discernment $\Theta_{i}$ of the associated Bf-constraint obtained by the Cartesian product of the involved variables domains, i.e., $\Theta_{i}=\left\{D_{i 1} \times \ldots \times D_{i k}\right\}$. The valuation $V_{i}$ is defined as follows:

$$
V_{i}=m_{i}: 2^{\Theta_{i}} \rightarrow[0,1] \mid \sum_{I \subseteq \Theta_{i}} m_{i}(I)=1
$$

Where $I$ is a singleton or a subset of instantiations. In the following, we shall restrict ourselves to normalized bbm distributions where $m_{i}(\emptyset)=0$.

If we may define an instantiation as a logical relation between variables, the finite amount of support (complete, partial or ignorant) enclosed in the bbm (i.e., $m_{i}(I)$ ) and derived from the available pieces of evidence can be interpreted as the potentiality degree of a given relation to, actually, occur so that the potentiality of the associated Bfconstraint to be satisfied by such variables instantiation(s), I. A second reading interprets this bbm distribution as preference levels induced over instantiations. Formally, let $\theta_{1}$ and $\theta_{2}$ two subsets of $\Theta_{i}\left(i . e ., \theta_{1}, \theta_{2} \subseteq \Theta_{i}\right), m\left(\theta_{1}\right)>$ $m\left(\theta_{2}\right)$ means that $\theta_{1}$ is more believable (certain) than $\theta_{2}$ and hence $\theta_{1}$ is, a priori, preferred to $\theta_{2}$ for the satisfaction of the given Bf-constraint. This latter reading shows that the bbm distribution has twofold purpose. The first is to quantify our belief about a given instantiation whereas the second is to induce a preorder, i.e., a priori preferences, among them.

The most appealing feature that makes the BFT an efficient tool is its faithfulness in recognition our knowledge as well as our ignorance. Obviously, the case of total knowledge matches the classical notion of perfect (certain and precise) relation where all tuples are known with certainty. This case is obtained through the certain belief function. Other kinds of relations may be modeled using the various functions offered by the BFT such as the vacuous belief function for uncertain and imprecise relation and the categorical belief function for certain but imprecise relation. 
Likewise, some operations may be applied on imperfect relations, such as the vacuous extension and the marginalization. The vacuous extension of an imperfect relation $R_{i}$ defined on $S_{i}$, to a larger set $S_{i}^{\prime}$, such that $S_{i} \subseteq S_{i}^{\prime}$, is an imperfect relation $R_{i}^{\left(\uparrow S_{i}^{\prime}\right)}$ defined on $S_{i}^{\prime}$ and obtained as follows:

$$
m_{i}^{\left(\uparrow S_{i}^{\prime}\right)}(\varphi)=\left\{\begin{array}{l}
m_{i}(\theta) \text { if } \varphi=\theta^{\uparrow S_{i}^{\prime}} \\
0 \text { otherwise }
\end{array} \text { for all } \theta \subseteq \Theta_{i}\right.
$$

Such that $\theta^{\uparrow S_{i}^{\prime}}$ denotes the cylindrical extension of the set $\theta$ to $S_{i}^{\prime}$. The vacuous extension is useful when we want to know to what extent a given instantiation may satisfy the Bf-constraint $C_{i}$. In fact, it corresponds to a refinement of knowledge.

The knowledge, initially, encapsulated in the bbm distribution can be refined as well as coarsened. The marginalization, which corresponds to a coarsening of knowledge, of an imperfect relation $R_{i}$ defined on $S_{i}$, to a coarser set $S_{i}^{\prime}$,i.e., $S_{i} \supseteq S_{i}^{\prime}$, is an imperfect relation $R_{i}^{\left(\downarrow S_{i}^{\prime}\right)}$ defined on $S_{i}^{\prime}$ and obtained as follows:

$$
m_{i}^{\left(\downarrow S_{i}^{\prime}\right)}(\varphi)=\sum_{\theta \subseteq \Theta_{i}: \theta^{\downarrow S_{i}^{\prime}}} m_{i}(\theta) \text { for all } \varphi \subseteq \Theta_{i}
$$

Such that $\theta^{\downarrow S_{i}^{\prime}}$ denotes the projection of the set $\theta$ to $S_{i}^{\prime}$. We can employ the marginalization when we want to know to what extent a given partial instantiation, if extended, may satisfy the Bf-constraint $C_{i}$.

\subsubsection{Prioritized Constraints}

In real world problems, not all the constraints are fully reliable. In fact, under uncertainty, the agent cannot entirely rely on some constraints that can be untrustworthy, misleading or that can be subject to change. For instance, in the agricultural production planning problem the reliability of some constraints depends on fluctuating prices and / or weather condition. In other words, some constraints may be relevant in a given circumstance but not in other circumstances. Regardless, this reliability affects the importance of each constraint to the set of solutions. For this reason, some constraints are prior to others.

To express priorities, we have recourse to the discounting principle provided by the BFT. First, we evaluate the reliability of each Bf-constraint $C_{i} \in C$ using a discounting factor $\theta$, so that, the smaller the reliability, the stronger the discounting. Second, we have to update the bbm distribution according to that factor. Let $m_{i}$ be a bbm distribution related to the Bf-constraint $C_{i}$ on the frame of discernment $\Theta_{i}$ and let $\left(1-\alpha_{i}\right)$ be the confidence degree allocated to that $\mathrm{Bf}$-constraint $C_{i}$ that corresponds to its priority level.
The updated bbm, denoted by $m_{i}^{\alpha}$ and induced from the old bbm $m_{i}$ discounted by the coefficient $\alpha_{i}$, where every lost mass is reassigned to the universe of discourse, is obtained as follows:

$$
m_{i}^{\alpha}(I)= \begin{cases}\left(1-\alpha_{i}\right) m_{i}(I) & \text { if } I \neq \Theta_{i} \\ \alpha_{i}+\left(1-\alpha_{i}\right) m_{i}(I) & \text { if } I=\Theta_{i}\end{cases}
$$

such that $\alpha_{i} \in[0,1]$, it is called the discounting factor which read as follows:

- $\alpha_{i}=0$ means that the Bf-constraint $C_{i}$ is fully reliable, so its priority is equal to 1 and the Bf-constraint is absolutely relevant. In this case, the bbm $m_{i}$ is left unchanged.

- $\alpha_{i}=1$ means that the reliability of the Bf-constraint $C_{i}$ is totally doubtful, so its priority will be equal to 0 , which means that it is possible to violate the Bfconstraint. In this case, all the information induced by the $\mathrm{Bf}$-constraint $C_{i}$ will be forthright discarded. The bbm $m_{i}$ becomes a vacuous function that corresponds to the total ignorance state (i.e., $m_{i}^{\alpha}\left(\Theta_{i}\right)=1$ ).

The priority determine the importance of the Bf-constraint. Let $C_{i}$ and $C_{j}$ be two Bf-constraints with priority levels, respectively, $\alpha_{i}$ and $\alpha_{j}$, if $\alpha_{i}<\alpha_{j}$ then the satisfaction of $C_{i}$ is more relevant than the satisfaction of $C_{j}$. The notion of priority induces a preorder over the Bf-constraints.

\subsubsection{Soft Constraints}

After expressing our beliefs on the imperfect relations and updating those beliefs using priorities, we shall extract the satisfaction degree of the Bf-constraints by each given instantiation $\theta$ in $\Theta$ aside using the pignistic probabilities produced by the TBM pignistic transformation [8] of the bbm distribution.

Let $C_{i}$ be a Bf-constraint, $R_{i}$ its relation and let $m_{i}$ be the associated bbm distribution over $\Theta_{i}$, the produced pignistic probability, denoted by $\operatorname{Bet} P_{i}$, is defined as follows:

$$
\operatorname{Bet}_{i}(\theta)=\sum_{\varphi \subseteq \Theta_{i}}\left(m_{i}(\varphi) \frac{|\theta \cap \varphi|}{|\varphi|}\right) \text {, for all } \theta \in \Theta_{i}
$$

where, $|\varphi|$ denotes the number of elements of $\varphi$. Hence, this notion of pignistic probability allows for expressing soft or flexible $\mathrm{Bf}$-constraints starting from imperfect relations. It is of interest to discern the difference between hard constraint that should be certainly and fully satisfied and soft constraint whose satisfaction is not required to be neither certain nor total. Therefore, the satisfaction of a given constraint becomes, essentially, a matter of degree, such that:

- $\operatorname{Bet}_{i}(\theta)=1$ means that the instantiation $\theta$ totally satisfies the $\mathrm{Bf}$-constraint $C_{i}$; 
- $\operatorname{Bet} P_{i}(\theta)=0$ means that the instantiation $\theta$ totally violates the Bf-constraint $C_{i}$;

- $0<\operatorname{Bet} P_{i}(\theta)<1$ means that the instantiation $\theta$ partially satisfies the Bf-constraint $C_{i}$;

A Bf-constraint $C_{i}$, whose scope is $S_{i}$, is said to be (totally or partially) satisfied by a given instantiation $\theta \in \Theta_{i}$, noted $\theta \models C_{i}$ if and only if $\operatorname{Bet}_{i}(\theta)>0$. A Bf-constraint $C_{i}$ is said to be unsatisfiable if there is no instantiation that satisfies it, i.e., $\forall \theta \in \Theta_{i}, \operatorname{Bet} P_{i}(\theta)=0$. Obviously, hard constraints are a particular case of $\mathrm{Bf}$-constraints which are satisfied only to 1 or 0 degree.

\subsubsection{Preferences over the solutions}

The $\operatorname{Bet} P$ also induces a preorder among instantiations. Formally, let $\theta$ and $\theta^{\prime}$ be two instantiations defined on the same set of variables, $\operatorname{Bet} P_{i}(\theta)>\operatorname{Bet} P_{i}\left(\theta^{\prime}\right)$ means that is, a posteriori, preferred to $\theta^{\prime}$ for the satisfaction of the soft Bf-constraint $C_{i}$. Hence the $\operatorname{Bet} P$ has twofold purpose as it allows expressing soft Bf-constraints and preferences among instantiations.

Classically, an instantiation $\theta$ of a set of variables $S \subseteq X$ is said to be consistent if and only if it satisfies all the constraints among that set. Within the BCSP view, the constraint satisfiability is not any more a yes/no query but a matter of degree and so the instantiation consistency is.

To get the consistency degree of an instantiation, we shall aggregate the satisfaction degrees of each $\mathrm{Bf}$ constraint by the instantiation under consideration. In the literature, several aggregation functions have been proposed, especially, for decision models where uncertainty and imprecision are key issues. In order to select the appropriate function, we have considered three imperative criteria. First, the aggregation function should fulfill the most basic consistency principle, every instantiation that totally violates (i.e., $\operatorname{Bet} P_{i}(\theta)=0$ ) at least one constraint is rejected. Hence, we need an aggregation operator that has an absorbent element $a=0$. Second, we require that the unit interval $[0,1]$ be closed to the sought for aggregation function, so that, the resulted values may be easily interpretable and comparable. Finally, in order to avoid falling into the same weakness as the Fuzzy and the Possibilistic CSPs that suffer from the "drowning effect" because of the egalitarian min-max operators use which barely discriminate between instantiations that satisfy the CSP to the same degree, we propose using a utilitarian operator for aggregation.

Given these criteria, we find out that the most appropriate function may be the geometric mean. Formally, the consistency degree of an instantiation $\theta$ of a set of variables
$S \subseteq X$ is obtained as follows:

$$
\begin{aligned}
\mathcal{C}(\theta) & =\operatorname{Bet}_{\wedge}\left\{R_{i} \mid S_{i} \subseteq S\right\}(\theta) \\
& =\left(\prod_{R_{i}^{\uparrow S} \mid S_{i} \subseteq S}\left\{\operatorname{Bet} P_{i}\right\}(\theta)\right)^{1 / k} \\
& =\left(\prod_{R_{i} \mid S_{i} \subseteq S}\left\{\operatorname{Bet} P_{i}\right\}\left(\theta^{\downarrow S}\right)\right)^{1 / k}
\end{aligned}
$$

such that, $k$ is the number of the $\mathrm{Bf}$-constraints covering $S$.

- If $\theta$ totally satisfies all the Bf-constraints covering $S$, it is said to be completely consistent, i.e., $\mathcal{C}(\theta)=1$.

- If $\theta$ totally violates, at least, one Bf-constraint is said to be inconsistent, i.e., $\mathcal{C}(\theta)=0$.

- Otherwise, it is said to be partially consistent, i.e.,

$$
0<\mathcal{C}(\theta)<1 \text {. }
$$

As the BCSP is a generalization of the classical model, if the relations are perfect, a given instantiation is either consistent (1) or inconsistent (0).

A solution of BCSP $(P)$ is every consistent complete instantiation $\theta$, i.e., an instantiation of all the variables in $X$ whose consistency degree is greater than 0 , so that, all the Bf-constraints in $C$ are satisfied. This consistency degree, evidently, corresponds to the satisfaction degree of the $\operatorname{BCSP}(P)$ by that instantiation.

$$
\mathcal{S}_{P}(\theta)=\mathcal{C}(\theta)=\left(\prod_{C_{i} \in C ; R_{i}^{\uparrow X}}\left\{\operatorname{Bet} P_{i}\right\}(\theta)\right)^{1 / m}
$$

such that, $m$ is the total number of the Bf-constraints covering $X$.

Accordingly, we can merely notice that the satisfaction degree of the BCSP, as defined above, accomplishes a sort of quantitative discrimination among the several instantiations inducing then a total preorder over them. Then, the higher is the satisfaction degree, the better is the instantiation.

The solution space of a BCSP $(P)$ of the set of all the feasible solutions, i.e.,

$$
\operatorname{Sols}(P)=\left\{\theta \in\left\{D_{1} \times \ldots \times D_{n}\right\} \mid \mathcal{S}_{P}(\theta)>0\right\}
$$

A BCSP $(\mathrm{P})$ is said to be:

- Totally consistent if and only if it has at least one solution that totally satisfies all the constraints of $\mathrm{C}$, i.e., $\exists \theta \in \operatorname{Sols}(P) \mid \mathcal{S}_{P}(\theta)=1$.

- Totally inconsistent if and only if all instantiations of $X$ are inconsistent, i.e., $\operatorname{Sols}(P)=\emptyset$ or also $\forall \theta \in\left\{D_{1} \times\right.$ $\left.\ldots \times D_{n}\right\} \mid \mathcal{S}_{P}(\theta)=0$. 
- Partially consistent if and only if all solutions are somehow feasible, i.e., $\operatorname{Sols}(P) \neq \emptyset \mid \forall \theta \in$ $\operatorname{Sols}(P), \mathcal{S}_{P}(\theta)<1$.

Toward the same view, the consistency degree of a BCSP is the satisfaction degree of its best solution, i.e.,

$$
\begin{aligned}
\mathbb{C}(P) & =\mathcal{S}_{P}\left(\theta^{*}\right) \\
& =\max _{\theta \in \operatorname{Sols}(P)}\left(\mathcal{S}_{P}(\theta)\right) \\
& =\max _{\theta \in \operatorname{Sols}(P)}\left(\prod_{C_{i} \in C ; R_{i}^{\uparrow X}}\left\{\operatorname{Bet} P_{i}\right\}(\theta)\right)^{1 / m}
\end{aligned}
$$

\section{A planning problem}

In this section, we suggest a Belief CSP model for a simple vegetable crops production planning problem under uncertainty. The problem is to decide which crop to plant in which plot (a measured area of land). However, vegetable crops are, generally, cost expensive and of uncertain profitability due to the fluctuating prices and its dependence to weather condition that affects the harvest yields. The generic problem could be the following: a number of crops must be produced in a number of plots $\left(a_{i}\right)$. Each plot has a limited area and grows one single crop. Each crop has a profit $\left(p_{j}\right)$ and a labor-hour $\left(h_{i}\right)$ per unit area $\left(1000 \mathrm{~m}^{2}\right)$ which are uncertain. The agriculturist's practical experience and his preferences are considered as pieces of evidence.

A farmer has to grow cucumber, pepper, potatoes, and peas in four plots $a_{1}, a_{2}, a_{3}$, and $a_{4}$. The total area of the land $(L)$ to be cultivated is $100.000 \mathrm{~m}^{2}(10 \mathrm{ha})$ where $a_{1}, a_{2}$, $a_{3}$, and $a_{4}$ represent, respectively, 40,30,20, and 10 percent of $L$. The farmer requires a minimum profit $(R)$ equal to 150.000 TND (Tunisian National Dinar) and a maximum labor-hour $(H)$ equal to 500 hours. As well, he prefers not to grow potatoes on the same plot for more than two years and after potatoes production, it is preferable to grow cleaning crops like cucumber to maintain the soil healthiness but he is doubtful about which crop he prefers more for $a_{1}$, pepper or peas. The evidence we have is that, last year, potatoes were grown in plot $a_{4}$.

A $\operatorname{BCSP}\left(X, D, C_{\alpha}, R\right)$ may be:

- $X=\left\{a_{1}, a_{2}, a_{3}, a_{4}\right\}$ is the set of four plots;

- $D=\left\{D_{1}, D_{2}, D_{3}, D_{4}\right\}$, where $D_{1}=D_{2}=D_{3}=$ $D_{4}=\left\{c u_{\left(p_{1}, h_{1}\right)}, p_{\left(p_{2}, h_{2}\right)}, p o_{\left(p_{3}, h_{3}\right)}, p e_{\left(p_{4}, h_{4}\right)}\right\}$ where $c u, p, p o$, and $p e$ stands respectively for cucumber, pepper, potatoes and peas;

- $C_{\alpha}=\left\{\left(C_{1}, \alpha_{1}\right),\left(C_{2}, \alpha_{2}\right),\left(C_{3}, \alpha_{3}\right)\right\}$ is a set of two
Bf-constraints.

$$
\begin{aligned}
& C_{1}: \sum_{i, j=1}^{n} a_{i} \cdot p_{j} \geq R ; \alpha_{1}=0.4 \\
& C_{2}: \sum_{i, j=1}^{n} a_{i} \cdot h_{j} \leq H ; \alpha_{2}=0.2 \\
& C_{3}: a_{i} \neq a_{k} \forall i, k=1 . .4 \text { and } i \neq k ; \alpha_{3}=0
\end{aligned}
$$

- $R=\left\{R_{1}, R_{2}, R_{3}\right\}$ is a set of imperfect relations associated to $C_{\alpha}$.

Giving the uncertain values of $p_{j}$ and $h_{j}$, the bbm distributions related to the imperfect relations $R_{1}$ and $R_{2}$ are, respectively, illustrated in Table 1.

The priority of the Bf-constraint $C_{3}$ is equal to 1(i.e., $1-\alpha_{3}$ ), so it is fully reliable and absolutely relevant which means that it should be certainly and fully satisfied. $C_{3}$ is a classical hard constraint. The associated relation $R_{3}$ is a certain but imprecise relation where there is more than single instantiation may fully satisfy the Bf-constraint $C_{3}$. $R_{3}$ is represented using the categorical bbm distribution $m_{3}$ as follows: $\forall \theta \subset \Theta_{3}$, if $a_{i} \neq a_{k} \forall i, k=1 . .4$ and $i \neq k$ then $\theta \in \varphi$ such that $m_{3}(\varphi)=1$, otherwise, $m_{3}(\theta)=0$. We can notice that $|\varphi|=4$ ! complete instantiations that fully satisfy $C_{3}$.

Table 1. The imperfect relations $R_{1}$ and $R_{2}$.

\begin{tabular}{|l||l|}
\hline$R_{i}$ & $m_{i}$ \\
\hline$R_{1}\left(\alpha_{1}=0.4\right)$ & $m_{1}\left\{\left(a_{1}, p\right),\left(a_{1}, p e\right)\right\}=0.5$ \\
& $m_{1}\left\{\left(a_{4}, p o\right)\right\}=0.3$ \\
& $m_{1}\left\{\left(a_{2}, c u\right),\left(a_{2}, p\right),\left(a_{3}, c u\right)\right\}=0.1$ \\
& $m_{1}\left\{\Theta_{1}\right\}=0.1$ \\
\hline$R_{2}\left(\alpha_{2}=0.2\right)$ & $m_{1}\left\{\left(a_{1}, c u\right),\left(a_{2}, c u\right)\right\}=0.3$ \\
& $m_{1}\left\{\left(a_{4}, c u\right)\right\}=0.4$ \\
& $m_{1}\left\{\left(a_{4}, p o\right),\left(a_{3}, p o\right)\right\}=0.1$ \\
& $m_{1}\left\{\Theta_{2}\right\}=0.2$ \\
\hline
\end{tabular}

Given the relative priorities, the updated bbm distributions $m_{1}^{\alpha}$ and $m_{2}^{\alpha}$ related, respectively, to the imperfect relations $R_{1}$ and $R_{2}$ are represented in Table 2. $R_{3}$ is left unchanged.

At this level, as our problem model is updated, we can compute the satisfaction degrees of each $\mathrm{Bf}$-constraint by any complete or partial instantiation $\theta$. For instance, let $\theta_{1}=\left\{\left(a_{1}, c u\right),\left(a_{2}, p\right)\right\}$ be a partial instantiation; the satisfaction degree of $C_{1}$ by $\theta_{1}$, i.e., $\operatorname{Bet}_{1}\left(\theta_{1}\right)=0.08$ whereas the satisfaction degree of $C_{2}$ by $\theta_{1}$, i.e., $\operatorname{Bet}_{2}\left(\theta_{1}\right)=0.17$. Let $\theta_{2}=\left\{\left(a_{1}, c u\right),\left(a_{2}, p\right),\left(a_{3}, p o\right),\left(a_{4}, p e\right)\right\}$ be a complete instantiation; $\operatorname{Bet} P_{1}\left(\theta_{2}\right)=0.32$ and $\operatorname{Bet}_{2}\left(\theta_{2}\right)=$ 0.25. If we get another complete instantiation $\theta_{3}=$ $\left\{\left(a_{1}, p e\right),\left(a_{2}, p\right),\left(a_{3}, p o\right),\left(a_{4}, c u\right)\right\} ; \operatorname{Bet} P_{1}\left(\theta_{3}\right)=0.47$ 
Table 2. The updated imperfect relations.

\begin{tabular}{|l||l|l|}
\hline$R_{i}$ & Priority & $m_{i}^{\alpha}$ \\
\hline$R_{1}$ & $1-\alpha_{1}$ & $m_{1}\left\{\left(a_{1}, p\right),\left(a_{1}, p e\right)\right\}=0.3$ \\
$\left(\alpha_{1}\right.$ & $=0.6$ & $m_{1}\left\{\left(a_{4}, p o\right)\right\}=0.18$ \\
$=0.4)$ & & $m_{1}\left\{\left(a_{2}, c u\right),\left(a_{2}, p\right)\right.$, \\
& & $\left.\left(a_{3}, c u\right)\right\}=0.06$ \\
& & $m_{1}\left\{\Theta_{1}\right\}=0.46$ \\
\hline$R_{2}$ & $1-\alpha_{2}$ & $m_{1}\left\{\left(a_{1}, c u\right),\left(a_{2}, c u\right)\right\}=0.24$ \\
$\left(\alpha_{2}\right.$ & $=0.8$ & $m_{1}\left\{\left(a_{4}, c u\right)\right\}=0.32$ \\
$=0.2)$ & & $m_{1}\left\{\left(a_{4}, p o\right),\left(a_{3}, p o\right)\right\}=0.08$ \\
& & $m_{1}\left\{\Theta_{2}\right\}=0.36$ \\
\hline
\end{tabular}

and $\operatorname{Bet} P_{2}\left(\theta_{3}\right)=0.45$. We can notice that the instantiation $\theta_{3}$ is preferred to $\theta_{2}$ for the satisfaction of both of the soft Bf-constraints $C_{1}$ and $C_{2}$. However, they are equally preferred for the satisfaction of $C_{3}$ as $\operatorname{Bet} P_{3}\left(\theta_{2}\right)=$ $\operatorname{Bet}_{3}\left(\theta_{3}\right)=0.04$.

Let us recall that every complete consistent instantiation is a possible solution for the BCSP. For example, one solution to the current BCSP may be $\theta_{2}=\left\{\left(a_{1}, c u\right),\left(a_{2}, p\right),\left(a_{3}, p o\right),\left(a_{4}, p e\right)\right\}$ whose consistency degree $\mathcal{C}\left(\theta_{2}\right)=0.15$, same as $\theta_{3}=$ $\left\{\left(a_{1}, p e\right),\left(a_{2}, p\right),\left(a_{3}, p o\right),\left(a_{4}, c u\right)\right\}$ whose consistency degree $\mathcal{C}\left(\theta_{3}\right)=0.2$. However, $\theta_{3}$ is preferred to $\theta_{2}$.

The best solution consistency degree is 0.2 . Hence, the current BCSP is partially consistent as its consistency degree, i.e., $\mathbb{C}(P)=0.2$.

In order to make our framework valid, as a first step, we have adapted the very basic Backtrack algorithm to solve the BCSPs. However, given the sound basis of the BCSP framework, other interesting solving algorithms as well as consistency ones may be easily extended.

\section{Further work}

The large-range expressivity brought by the BFT allows for covering both aspects of imperfection, uncertainty as well as imprecision, soft and prioritized constraints, and preferences over solutions. We may consider the BCSP as a unifying and general CSP framework where mapping to other frameworks is possible using the different kinds of relations. In addition, the BCSP is too close to the real world problems where an agent is not required to provide prior information and it takes into account his available knowledge and his preferences.

As a generalization of the standard CSP framework, the classical algorithms (e.g., the branch and bound algorithm, the consistency algorithms) can be adapted to our BCSP framework. Currently, we are working on implementing a specific algorithm for the BCSP employing some measures such as the belief and the plausibility offered by the BFT and discussing its complexity.

\section{References}

[1] Arthur P. Dempster. Upper and lower probabilities induced by a multivalued mapping. The Annals of Mathematical Statistics, 1967, 38(2):325-339.

[2] Didier Dubois, Helene Fargier, and Henri Prade. Possibility theory in constraint satisfaction problems: Handling priority, preference and uncertainty. Applied Intelligence, 1996, 6:287-309.

[3] Helene Fargier, Jerome Lang, and Thomas Schiex. Selecting preferred solutions in fuzzy constraint satisfaction problems. In Proc. of the first European Congress on Fuzzy and Intelligent Technologies, 1993, 3: 11281134.

[4] Helene Fargier and Jerome Lang. Uncertainty in constraint satisfaction problems: a probabilistic approach. In Proc. of the Second European Conference on Symbolic and Quantitative Approaches to Reasoning and Uncertainty, 1993, 747:97-104.

[5] Alan K. Mackworth. Consistency in networks of relations. Artificial Intelligence, 1977, 8(1):99-118.

[6] Ugo Montanari. Networks of constraints: Fundamental properties and applications to picture processing. Information Sciences, 1974,7(2):95-132.

[7] Glenn Shafer. A Mathematical Theory of Evidence. Princeton University Press, Princeton, NJ, 1976.

[8] Philippe Smets. Belief functions. Non-Standard Logics for Automated Reasoning, 1988, 253-286.

[9] Philippe Smets. Imperfect information: Imprecision Uncertainty. Uncertainty Management in Information Systems. From Needs to Solutions, 1997, 225-254.

[10] Thomas Schiex. Possibilistic constraint satisfaction problems or How to handle soft constraints?. In Proc. of the 8th International Conference on Uncertainty in Artificial Intelligence, 1992, 268-275. 